\title{
A risk assessment method based on the failure analysis of medical devices in the adult Intensive Care Unit
}

\author{
By Fabiola M. Martinez-Licona, Sergio E. Perez-Ramos
}

Electrical Engineering Department, Universidad Autonoma Metropolitana, Mexico City

\begin{abstract}
Backgrounds and Objective: The Intensive Care Unit (ICU) receives patients whose situation demands high complexity tasks. Their recovery depends on medical care, their response to medications and clinical procedures, and the optimal functioning of the medical devices devoted to them. Adverse events in the ICU due to failures in the facilities, particularly medical devices, impact the patients, operators, and all involved in their care. The origins of the technological failures seem to be more oriented to the interaction between the equipment and the operator. Once the medical equipment is functioning, we must guarantee its correct execution to meet both the clinical service's objectives and the expectations of those involved in care, including the patients themselves. We present an approach to quality management based on failure analysis as the source of risk for medical devices' functioning and operation in the ICU. We decided to address it through a systematic approach by using elements from the Failure Mode and Effects Analysis (FMEA) method and the Ishikawa diagrams' support to obtain the causes graphically.

Material and Methods: We used the risk analysis framework as a basis of the methodology. By obtaining the causes and sub causes of technological failures in the ICU for adult patients, we adapted some of the FMEA method and applied the Ishikawa diagrams to analyze the relationship between cause and failure. The ICU devices came from the Official Mexican Standard and the World Health Organization (WHO) information related to the ICU operation and facilities. The data from the causes of failure came from specialized consultation and discussion forums on medical devices where these topics were addressed; we searched for over five years in Spanish forums. We proposed a calculation of the Risk Priority Number based on the information subtracted from the forums. Then, we defined an indicator showing the priority level used to address the issue.

Results: In general, the results showed that most of the medical equipment failure causes have medium and high-risk priority levels and, in some cases, the cause presented as the most prevalent did not match with the reported in official documents such as technical or operation manuals. The most frequent causes found are related to electrical system issues and operation skills. We presented three study cases: defibrillator, vital sign monitor, and volumetric ventilator, to show the risk level designation. The conclusions inferred from these cases are oriented to training strategies and the development of support material in Spanish.

Conclusion: The development of risk management methodologies to monitor and solve potential hazard situations in critical areas is valuable to the health technology management program. The FMEA method showed a solid basis for the risk assessment processes, and its application to the ICU medical technology allowed the creation of the evidence supporting the decision-making process concerning strategic solutions to guarantee patient safety.
\end{abstract}

Keywords - Risk Assessment, Failure analysis, FMEA, ICU medical equipment, Health Technology Management.

Copyright (C) 2021. This is an open-access article distributed under the terms of the Creative Commons Attribution License (CC BY): Creative Commons - Attribution 4.0 International - CC BY 4.0. The use, distribution or reproduction in other forums is permitted, provided the original author(s) and the copyright owner(s) are credited and that the original publication in this journal is cited, in accordance with accepted academic practice. No use, distribution or reproduction is permitted which does not comply with these terms. 


\section{INTRODUCTION}

The patients in the Intensive Care Unit (ICU) are under unique circumstances. This situation is due to the requirement of specialized multi-organ support actions needed to restore their health, including medical devices. ${ }^{1}$ Furthermore, the ICU patients' complexity makes them depend on medical care, their response to medications and clinical procedures, and the optimal functioning of the medical devices devoted to them. Studies carried out in this regard have shown the impact of failures related to technology and its applications in the analysis of adverse events at ICU, ${ }^{2}$ and the importance of safety improvement in using medical devices in this area to have greater control of incidents. ${ }^{3}$

Technological failure is defined in the hospital environment as an event in which medical equipment has stopped working correctly, which is associated with a probability of harming the patient or the operator. ${ }^{4}$ The origin of technological failures can be approached from different perspectives; some of the most relevant has to do with the negative effect on the patient or the context of medical devices' operation. Some sources of failure that have been identified in this regard are ${ }^{5}$ :

- The wrong dynamic range of measurement

- A lack of training in the operation of the equipment

- A lack of quality control in measurements

- A lack of quality control in pre-installations

- The wrong design

The origins of the technological failures mentioned are more oriented to the interaction between the equipment and the operator. Once the equipment is in operation, we must guarantee its correct execution to meet both the clinical service's objectives and the expectations of those involved in care, including the patients themselves. In this sense, technological failure becomes a reference point for developing plans and strategies that help improve quality. Within this frame of reference, aspects related to the medical device's operation will be taken as a quality feature so that the failure analysis approaches from a strictly technological perspective. Furthermore, it implies that the factors associated with its operation and functioning, including infrastructure, device design, and human resources, will be analyzed around the medical device and not as independent causes. Thus, we can design strategies for the containment and eventual elimination of the fault.

As for the ICU, analyzing the causes of medical devices' failures will make it possible to develop plans for risk management and control of related incidents. This kind of management process is particularly relevant, given the vulnerable condition of the patients treated in ICU demands actions that guarantee their safety and those who interact with them. We must address two considerations: a failure may be due to more than one factor, and in ICU, the potential factors involved increase the difficulty of the analysis. We decided to address the issue through a systematic approach, so we chose to take the Failure Mode and Effects Analysis (FMEA) method.

FMEA method is a systematic process that identifies the potential product or process design failures before they occur to eliminate them or minimize the associated risk. ${ }^{6}$ Although this method has been used more frequently in the automotive industry; it can detect and contain potential failures in various natures' products and processes. Therefore, its application to the medical field has been growing. ${ }^{7}$ It includes improving patient safety, ${ }^{8}$ the analysis of risk points in the implementation of smart devices, ${ }^{9}$ its application in radiotherapy, ${ }^{10}$ or quality management in the clinical laboratory. ${ }^{11}$ FMEA method integrates two stages for its implementation: identifying the failure and its evaluation. ${ }^{12}$

The identification stage includes the following: the process' phases list to be analyzed, the potential failure modes, the identification of the effects, if the failure mode occurred, the causes that could have originated them, and the discovery of the controls that the process has to prevent failures from happening, that is, prevention and detection. The evaluation stage evaluates the severity, occurrence, and detection of the failure and identifies the points to apply corrective and improvement actions. Finally, the stage is complemented by assigning risk through an indicator and prioritizing failure modes to take action.

Tools such as Pareto diagrams or cause-effect diagrams are common to identify the causes of failures. In this case, we selected the Ishikawa diagram because it facilitates analyzing problems and solutions in aspects such as quality of processes, products, and services. The Ishikawa diagrams rely on a logical order to structure the information 
and take the form of a fishbone; the multiple cause and effect relationships of the variables that intervene in the processes are presented. ${ }^{13}$

We present an approach to quality management that starts from analyzing failures as sources of risk for medical devices' functioning and operation in the ICU. From the FMEA, we used the first stage, identification of the failure. Then, with the Ishikawa diagrams' support, we obtained graphic displays of its causes and origins where created, which, together with a numerical indicator, allowed determining the prioritization of corrective actions.

\section{METHODS}

The methodology we used was founded on the risk analysis framework. By considering the technological failures that occur in the ICU for adult patients, we identified their causes and sub-causes. We adapted some elements from the FMEA method with the support of Ishikawa diagrams to analyze the relationship between cause and failure.

We obtained the ICU medical devices' identification to be analyzed from the Official Mexican Standard NOM025-SSA3-2013 for the organization and operation of intensive care units ${ }^{14}$ and the central medical equipment section of the World Health Organization (WHO). ${ }^{15}$ Then, for each piece of medical equipment, the causes of failure were classified into the following five categories ${ }^{13}$ and incorporated into an Ishikawa diagram:

- Human resources that are involved in the operation

- Environment or conditions under which medical equipment operates

- Materials used for its operation

- Methods for the development of functions for its operation

- Machines or any equipment or tools required to perform the work

We obtained the information to identify the possible causes of failures associated with using the equipment from the search in specialized consultation and discussion forums on medical devices where these topics were addressed; we searched for over five years in Spanish forums. Among the platforms consulted are yoreparo. com, ayudabiomedica.com, forumsdeelectronica.com, sefh. es, and elhospital.com. The information was completed by consulting the equipment's technical and operation manuals. We identified the possible causes of a failure for each medical equipment and, based on the number of mentions found in the forums consulted, their priority level. We determined to evaluate the quality of operation of each piece of equipment by defining the following metric based on the Risk Priority Number (RPN) that, according to the FMEA evaluation stage, is defined by

\section{RPN= Severity*Occurrence*Detection (1)}

Each of the terms included in (1) were adapted to the forum information context, so:

- Severity is computed by taking the number of found mentions per specific cause divided by the highest value of mentions per any cause in the medical device.

- Occurrence is calculated by taking the number of mentions per specific cause divided by the total number of mentions in the medical device.

- For Detection we proposed to assign three levels of impact of the failure, 1 to low, 2 to medium, and 3 to high, according to the information reported in the equipment's medical manuals. We carried out the assignment by searching the troubleshooting sections for the frequency of reported failures and solutions, assigning a higher level to the most frequent.

Then, we defined an indicator that shows a level of priority that can be used to address the issue. First, we normalized the RPN for each failure cause in the equipment (RPNn); then, we classified it into low, medium, and high categories based on the proportion of the RPNn failure cause within the related equipment. In general, high class was assigned to RPNn $>0.5$, low priority to RPNn $<0.1$, and medium priority to RPNn between these two values. Following, we proposed a priority level percentage indicator that shows the general situation of the equipment as:

\%_Priority_Level = (Number of mentions per RPN class $/$ number of total mentions per equipment)*100

We determined each medical equipment's risk level based on the percentage of failures with high and medium priority levels obtained from the priority indicator. These 
results enabled us to focus on specific issues to develop action plans to address them.

\section{RESULTS}

The ICU medical equipment included in the analysis were:

- Apnea monitor

- Central monitoring
- Defibrillator

- Hospitalization bed

- Infusion pump

- Portable X-ray system

- Vital sign monitor

- Volumetric ventilator

The numbers of causes found, total mentions identified, and percentages of priority levels for each one are shown in Table 1.

TABLE 1. Causes, Mentions, Priority Levels (PL) Percentage Found in ICU Medical Devices

\begin{tabular}{|c|c|c|c|c|c|}
\hline Medical Device & Causes & Total Mentions & \% High PL. & \% Medium PL. & \% Low PL. \\
\hline Apnea Monitor & 11 & 24 & 50 & 33.33 & 16.67 \\
\hline Central Monitoring & 10 & 24 & 62.50 & 25 & 12.50 \\
\hline Defibrillator & 14 & 39 & 53.85 & 33.33 & 12.82 \\
\hline Hospitalization Bed & 13 & 34 & 26.47 & 44.12 & 29.41 \\
\hline Infusion Pump & 14 & 55 & 49.09 & 21.82 & 29.09 \\
\hline Portable X-ray & 17 & 69 & 39.13 & 23.19 & 37.68 \\
\hline Vital Signs Monitor & 15 & 39 & 46.15 & 38.46 & 15.38 \\
\hline Volumetric Ventilator & 13 & 38 & 28.95 & 44.74 & 26.32 \\
\hline
\end{tabular}

We present below the Defibrillator, Vital Signs Monitor, and Volumetric Ventilator cases to illustrate the results.

\section{Case 1: Defibrillator:}

Table 2 shows the causes found, the number of mentions, the values of severity, occurrence, and detection obtained, and the computed normalized RPN. The cause with the highest number of mentions was the suspension of the electrical system with 5. In contrast, the causes with the lowest number of mentions were related to the equipment's documentation. The prioritization was carried out as follows:

- Low priority: $0.01 \leq \mathrm{RPN}_{\mathrm{n}} \leq 0.1$

- Medium priority: $0.16 \leq \mathrm{RPN}_{\mathrm{n}} \leq 0.24$

- High priority: $0.43 \leq \mathrm{RPN}_{\mathrm{n}} \leq 1$

The last column's color corresponds to the priority class assigned, green to low, yellow to medium, and red to high priority level.
To illustrate obtaining the Severity, Occurrence, Detection and $\mathrm{RPN}_{\mathrm{n}}$ values, we will take the failure cause "Insufficient battery charge" (ibc).

$$
\begin{aligned}
& \text { Severity }_{i b c}=\frac{\text { Mentions }_{i b c}}{\text { Highest Mention Number }} \text { defibrillator }=\frac{4}{5}=0.8 \\
& \text { Occurrence }_{i b c}=\frac{\text { Mentions }_{i b c}}{\text { Total Mention Number }} \text { defibrillator }_{39}=\frac{4}{39}=0.102 \\
& \text { Detection }_{i b c}=3(\text { It was found in all the manuals consulted })
\end{aligned}
$$

Then, we get the $\mathrm{RPN}_{\mathrm{n}}$ :

$R P N_{n_{-} i h c}=\frac{\text { Severity }_{i b c} * \text { Occurence }_{i b c} * \text { Detection }_{i b c}}{\text { Max_RPN } N_{\text {defibrillato } r} r}=\frac{0.8 * 0.102 * 3}{0.3846}=0.6365 \approx 0.64$ 
TABLE 2. Causes, Mentions, RPNn Data, and Priority Classes Found in the Defibrillator

\begin{tabular}{|c|c|c|c|c|c|}
\hline Cause found & No. Mentions & Severity & Occurrence & Detection & RPNn \\
\hline Emergency electrical system & 5 & 1 & 0.128 & 3 & $\mathbf{1}$ \\
\hline Suspension of electrical service & 4 & 0.8 & 0.102 & 3 & $\mathbf{0 . 6 4}$ \\
\hline Insufficient battery charge & 4 & 0.8 & 0.102 & 3 & $\mathbf{0 . 6 4}$ \\
\hline Difficulty using the defibrillator & 4 & 0.8 & 0.102 & 3 & $\mathbf{0 . 6 4}$ \\
\hline Broken electrode cables & 4 & 0.8 & 0.102 & 2 & $\mathbf{0 . 4 3}$ \\
\hline Transients caused by other loads with the same supply & 3 & 0.6 & 0.076 & 2 & 0.24 \\
\hline Voltage fluctuations & 3 & 0.6 & 0.076 & 2 & 0.24 \\
\hline Power cord & 3 & 0.6 & 0.076 & 1 & 0.12 \\
\hline Poor electrode cleaning or incorrect application & 2 & 0.4 & 0.051 & 3 & 0.16 \\
\hline Battery life & 2 & 0.4 & 0.051 & 2 & $\mathbf{0 . 1 1}$ \\
\hline Faulty electrodes & 2 & 0.4 & 0.051 & 3 & 0.16 \\
\hline Incorrect electrode placement & 1 & 0.2 & 0.025 & 2 & $\mathbf{0 . 0 3}$ \\
\hline Lack of manuals and/or guides & 1 & 0.2 & 0.025 & 1 & $\mathbf{0 . 0 1}$ \\
\hline Difficulty with the documents language & 1 & 0.2 & 0.025 & 1 & $\mathbf{0 . 0 1}$ \\
\hline
\end{tabular}

The Max_RPNdefibrillator was obtained from computing severity, occurrence, and detection of the failure cause with most mentions (Emergency electrical system). Since $\mathrm{RPN}_{\mathrm{n} \_ \text {ibc }}>0.5$, the priority for this failure cause is high, so it gets the red color.
Next, we elaborated the Ishikawa diagram, shown in Figure 1, where the causes of failures were located in the established categories based on their failure impact, the most significant impact near the fishbone. The colors indicate the assigned priority level: red-high, yellowmedium, and green-low.

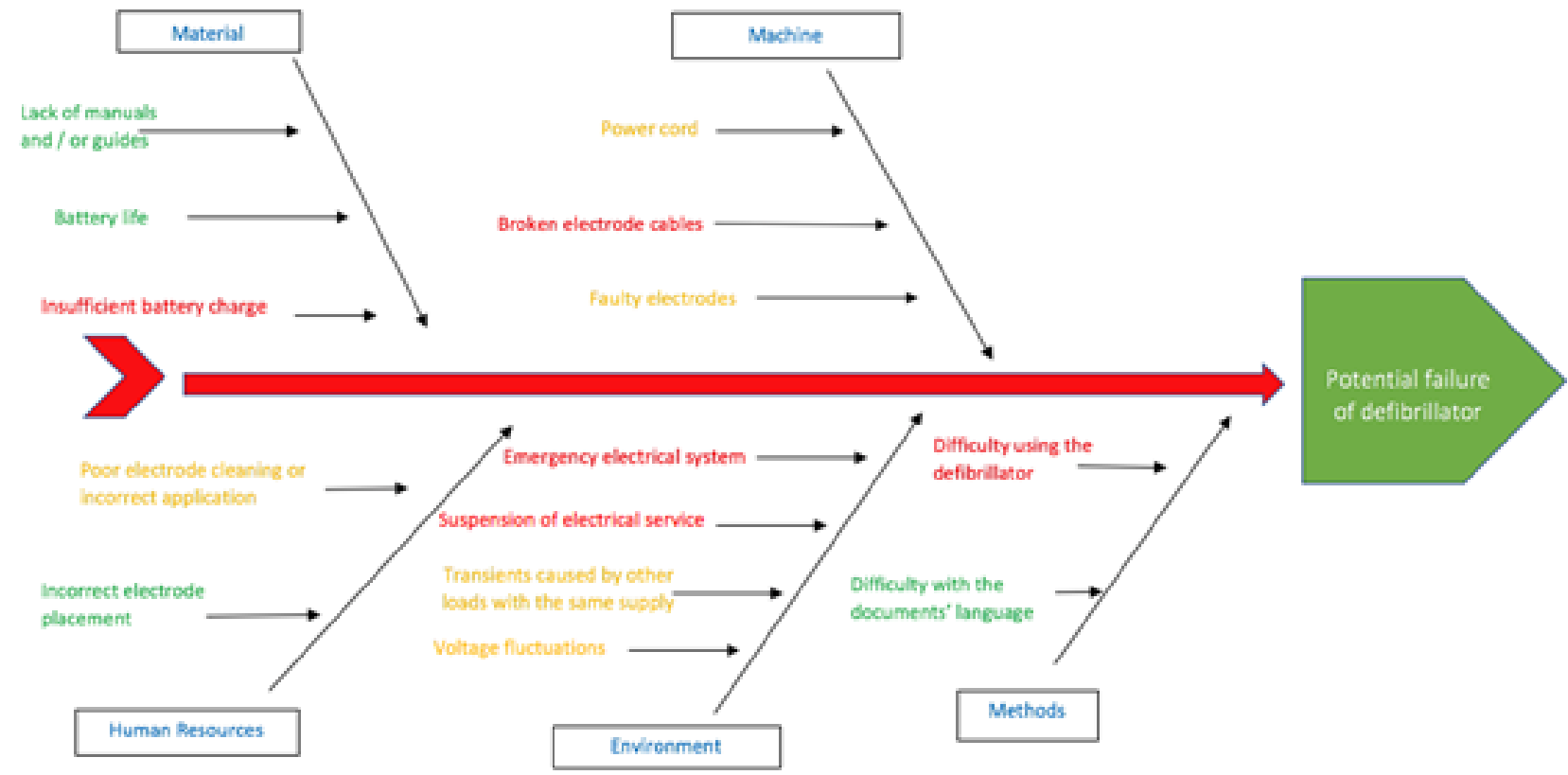

FIGURE 1. Ishikawa diagram for the defibrillator failure analysis. 


\section{Case 2: Vital Signs Monitor:}

Table 3 shows the causes found, the number of mentions, the values of severity, occurrence, and detection obtained, and the computed normalized RPN. For example, the cause with the highest number of mentions was related to the emergency electrical system with 6 . On the other hand, the causes with the lowest number of mentions included electrical supply and equipment documentation issues. In this case, the prioritization was carried out as follows:

- Low priority: $0.03 \leq \mathrm{RPN}_{\mathrm{n}} \leq 0.06$

- Medium priority: $0.11 \leq \mathrm{RPN}_{\mathrm{n}} \leq 0.25$

- High priority: $0.35 \leq \mathrm{RPN}_{\mathrm{n}} \leq 1$
As in the previous case, the last column's color corresponds to the priority class assigned, green to low, yellow to medium, and red to high priority. We elaborated the Ishikawa diagram corresponding to this medical equipment, shown in Figure 2, using the same settings for the previous case of the failure location and the color assigned according to the priority.

We elaborated the Ishikawa diagram corresponding to this medical equipment, shown in Figure 2, using the same settings for the previous case of the failure location and the color assigned according to the priority.

TABLE 3. Causes, Mentions, RPN Data and Priority Classes Found in the Vital Signs Monitor

\begin{tabular}{|c|c|c|c|c|c|}
\hline Cause found & No. Mentions & Severity & Occurrence & Detection & Norm. RPN \\
\hline Emergency electrical system & 6 & 1 & 0.153 & 2 & 1 \\
\hline Suspension of electrical service & 5 & 0.833 & 0.128 & 1 & 0.35 \\
\hline Power cord & 4 & 0.666 & 0.102 & 3 & 0.67 \\
\hline Difficulty using the monitor & 3 & 0.5 & 0.076 & 3 & 0.38 \\
\hline Insufficient backup batteries & 3 & 0.5 & 0.076 & 2 & 0.25 \\
\hline $\begin{array}{l}\text { Communication with the non-invasive pressure module } \\
\text { sensor }\end{array}$ & 3 & 0.5 & 0.076 & 2 & 0.25 \\
\hline Battery charge timeout & 3 & 0.5 & 0.076 & 1 & 0.13 \\
\hline $\begin{array}{l}\text { Lack of knowledge of the use of the control console for } \\
\text { calibration and adjustment (software) }\end{array}$ & 2 & 0.333 & 0.051 & 3 & 0.17 \\
\hline Communication with the heart rate module & 2 & 0.333 & 0.051 & 3 & 0.17 \\
\hline Communication with the pulse oximetry module & 2 & 0.333 & 0.051 & 2 & 0.11 \\
\hline Communication with the temperature module & 2 & 0.333 & 0.051 & 1 & 0.06 \\
\hline Voltage fluctuations & 1 & 0.166 & 0.025 & 3 & 0.04 \\
\hline Lack of manuals and / or guides & 1 & 0.166 & 0.025 & 3 & 0.04 \\
\hline Transients caused by other loads with the same supply & 1 & 0.166 & 0.025 & 2 & 0.03 \\
\hline Difficulty with the documents' language & 1 & 0.166 & 0.025 & 2 & 0.03 \\
\hline
\end{tabular}

Table 4 shows the causes found, the number of mentions, the values of severity, occurrence, and detection obtained, and the computed normalized RPN. In this equipment, the cause with the highest number of mentions was related to the power cord with 6 . On the other hand, there were only two causes with the lowest number of mentions, including electrical transients and pneumatic systems. In this case, the prioritization was carried out as follows:

- Low priority: $0.01 \leq \mathrm{RPN}_{\mathrm{n}} \leq 0.17$

- Medium priority: $0.22 \leq \mathrm{RPN}_{\mathrm{n}} \leq 0.38$

- High priority: $0.69 \leq \mathrm{RPN}_{\mathrm{n}} \leq 1$ 


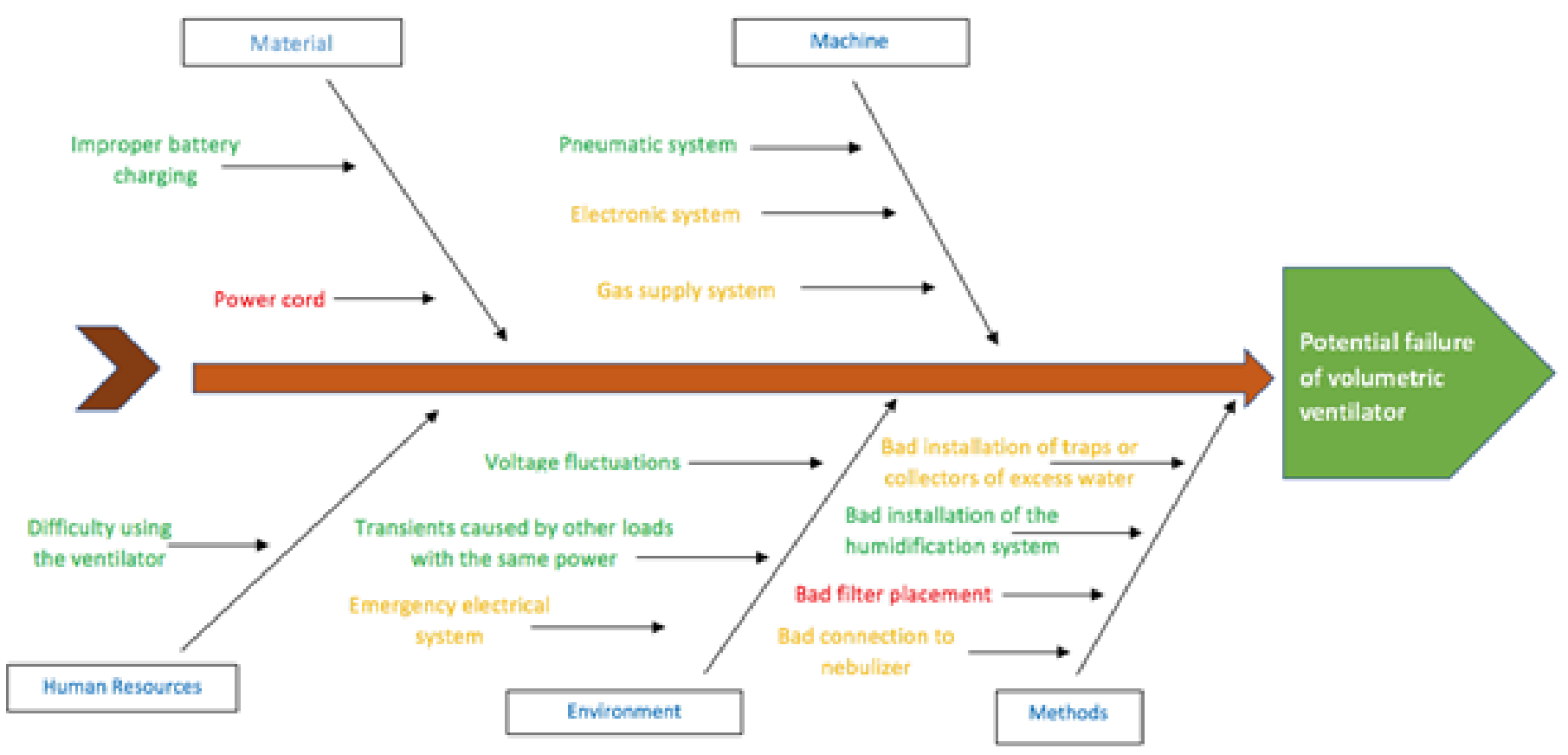

FIGURE 2. Volumetric Ventilator

TABLE 4. Causes, Mentions, RPNn Data and Priority Classes Found in the Volumetric Ventilator

\begin{tabular}{|c|c|c|c|c|c|}
\hline Cause found & No. Mentions & Severity & Occurrence & Detection & Norm. RPN \\
\hline Power cord & 6 & 1 & 0.157 & 2 & 1 \\
\hline Bad filter placement & 5 & 0.833 & 0.131 & 2 & $\mathbf{0 . 6 9}$ \\
\hline Bad connection to nebulizer & 4 & 0.666 & 0.105 & 1 & 0.22 \\
\hline Emergency electrical system & 4 & 0.666 & 0.105 & 1 & 0.22 \\
\hline Bad installation of traps or collectors of excess water & 3 & 0.5 & 0.078 & 3 & 0.38 \\
\hline Gas supply system & 3 & 0.5 & 0.078 & 3 & 0.38 \\
\hline Electronic system & 3 & 0.5 & 0.078 & 2 & 0.25 \\
\hline Bad installation of the humidification system & 2 & 0.333 & 0.052 & 3 & $\mathbf{0 . 1 7}$ \\
\hline Improper battery charging & 2 & 0.333 & 0.052 & 3 & $\mathbf{0 . 1 7}$ \\
\hline Voltage fluctuations & 2 & 0.333 & 0.052 & 2 & $\mathbf{0 . 1 1}$ \\
\hline Difficulty using the ventilator & 2 & 0.333 & 0.052 & 2 & $\mathbf{0 . 1 1}$ \\
\hline Transients caused by other loads with the same power & 1 & 0.166 & 0.026 & 3 & $\mathbf{0 . 0 4}$ \\
\hline Pneumatic system & 1 & 0.166 & 0.026 & 1 & $\mathbf{0 . 0 1}$ \\
\hline
\end{tabular}

The last column shows the priority class assigned: green to low, yellow to medium, and red to high priority level following the color agreement.
In the same way, we elaborated the Ishikawa diagram corresponding to this medical equipment, shown in Figure 3, using the same priority color assignment of the other cases. 


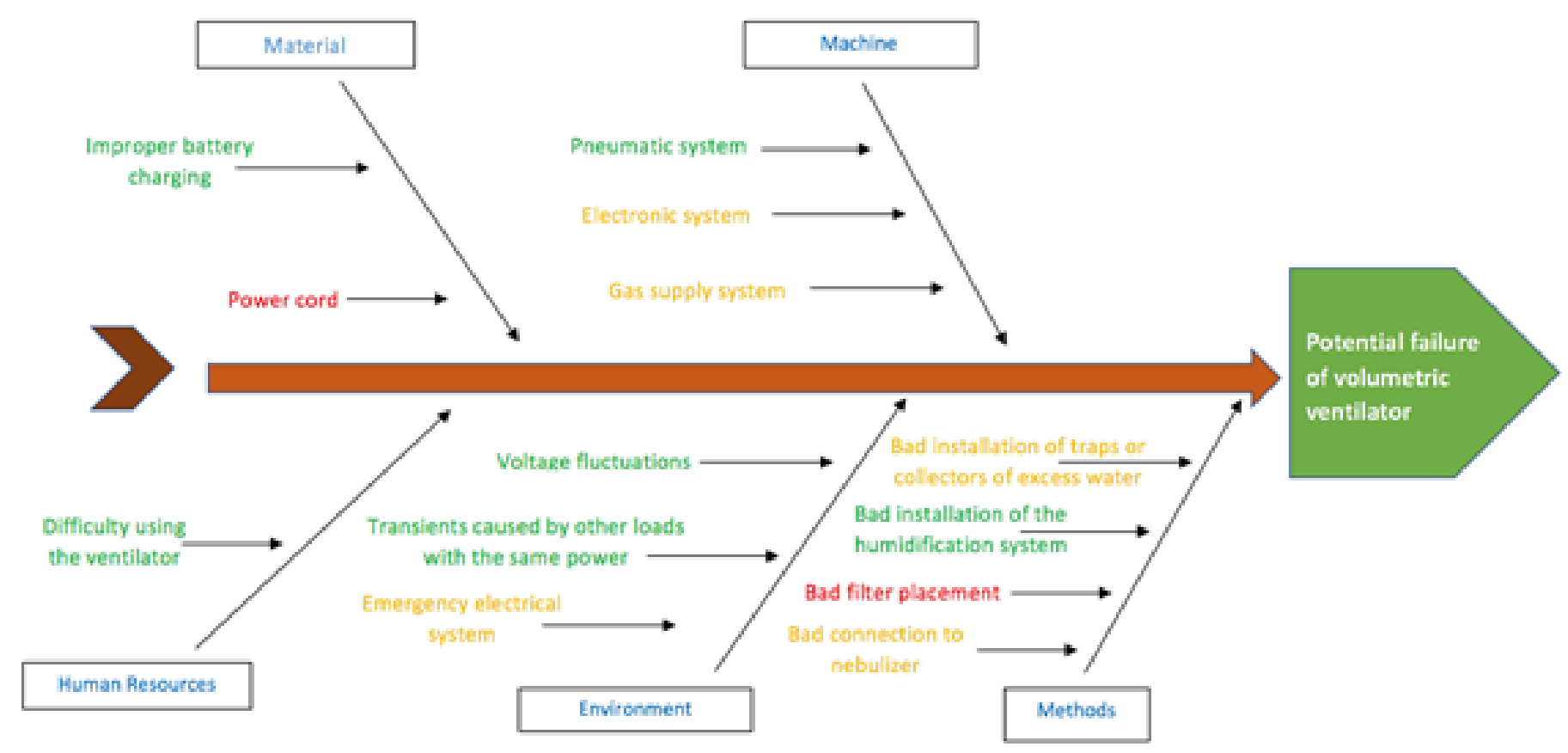

FIGURE 3. Ishikawa diagram for the volumetric ventilator failure analysis. Modified from ${ }^{19}$

Table 5 shows the failures that occurred most frequently in the remaining ICU medical equipment's consultation forums and Table 6 shows the risk level assigned after considering the priority levels obtained from the analysis.

The performance of each piece of equipment was obtained from considering the high and medium priority levels. Accordingly, we established the following Risk Levels:

- High: $75 \leq$ PL $\leq 100$. The equipment's operation presents failures that must be addressed immediately since its impact directly affects the patient's condition. The actions to be taken must be a priority so that the failure does not cause a significant problem.

- Medium: $51 \leq \mathrm{PL} \leq 74$. The equipment generally works as expected, but some elements indicate that a failure could compromise the performance and have consequences for patient care.

- Low: $0 \leq$ PL $\leq 50$. The equipment works ideally or closely. Care measures should focus on maintaining and improving its functioning to have the level of risk under control.
TABLE 5. Most Frequent Failure Causes in the ICU Medical Equipment

\begin{tabular}{|c|c|}
\hline Failure & Medical Equipment \\
\hline Suspension of electrical service & Apnea Monitor \\
\hline Emergency electrical system & Apnea Monitor \\
\hline Suspension of electrical service & Central Monitoring \\
\hline Emergency electrical system & Central Monitoring \\
\hline Wear of controls, handles and knobs & Hospitalization Bed \\
\hline Connections box & Hospitalization Bed \\
\hline $\begin{array}{l}\text { Difficulty using the hospitalization } \\
\text { bed }\end{array}$ & Hospitalization Bed \\
\hline $\begin{array}{l}\text { Lack of knowledge in the use of the } \\
\text { control, calibration and adjustment } \\
\text { system (software) }\end{array}$ & Infusion Pump \\
\hline Emergency electrical system & Infusion Pump \\
\hline Voltage fluctuations & Infusion Pump \\
\hline Hold alarm & Infusion Pump \\
\hline Emergency electrical system & Portable X-ray system \\
\hline Overheating & Portable X-ray system \\
\hline Inactivity & Portable X-ray system \\
\hline Suspension of electrical service & Portable X-ray system \\
\hline Inefficient equipment placement & Portable X-ray system \\
\hline
\end{tabular}


TABLE 6. Risk Level Assigned to the ICU Medical Equipment

\begin{tabular}{|c|c|c|}
\hline Medical Device & PL High+ Medium & Risk Level \\
\hline Apnea Monitor & 83.33 & High \\
\hline Central Monitoring & 87.5 & High \\
\hline Defibrillator & 87.18 & High \\
\hline Hospitalization Bed & 70.59 & Medium \\
\hline Infusion Pump & 70.91 & Medium \\
\hline Portable X-ray & 62.32 & Medium \\
\hline Vital Signs Monitor & 84.61 & High \\
\hline Volumetric Ventilator & 73.69 & Medium \\
\hline
\end{tabular}

\section{DISCUSSION}

Risk management is a crucial element for the efficient management of medical technology. Unfortunately, its principles and applications in this area have not yet reached a desired level of consolidation, so work on this issue should be promoted. In a critical area, the risk management repercussions impact both the patient and the operator, even reaching the infrastructure due to the complicated relationship these components have for functioning. Failure analysis is an approach to risk management that allows evaluating quality, in this case, reflected in operation, and provides elements that can be integrated with those used to manage medical equipment, such as schedules and maintenance reports, preventive or corrective maintenance documents. These components provide relevant information, but it is their systematic and well-documented integration that adds value for the development of action plans to control these failures.

The FMEA method has proven to be a practical analytical element in different settings. At ICU, it has been applied to evaluate clinical aspects such as pressure injury due to critical illness combined with interventions and therapies. ${ }^{16}$ When adapting some of the FMEA components to the information obtained from the ICU's equipment, it is possible to analyze the interaction between the different natures of the causes that generate failures. The Ishikawa diagrams complemented the analysis by contrasting the practical occurrence of the failures reported in the forums, with which they report the technical and operation manuals, which we would consider as more formal and official documentation.
The consultation and discussion forums are a source that may not offer high reliability compared to the information obtained from more official and formal documentation such as technical or operating manuals. However, their presence in the community shows a practical reality of the failures that appear in the engineers' and technicians' daily actions and technicians in charge of attending to the equipment considered; it also represents a need to share and communicate problems and solutions at a higher level of specificity. The quality of this source depends on the seriousness with which the community presents the cases and their responses; we assume this requirement is met given these forums' purpose.

The results show that in practice, ICU medical equipment requires constant and detailed care. None of the equipment was considered obtained at a low-risk level; this implies a constant presence of risk that may come from different sources. Portable X-ray is the device with the lowest risk level; although it is not continuously used, its most prevalent causes are related to infrastructure and operating conditions. In contrast, the monitoring devices are the ones that obtained a higher score in the level of risk; problems associated with the quality of the electrical system are more prevalent as causes of failure. In the case of the vital signs monitor (case 2), it is striking that the difficulty in its use has a high priority. The lack of knowledge of the control console's use for calibration and adjustment (software) has medium priority, and the lack of manuals and documentation has low priority. The location of these causes in the Ishikawa diagram indicates that they have a high impact on the occurrence of potential equipment failure. With this information, a latent training need and the development of support material in Spanish for staff can be inferred.

The defibrillator is another device that scored high risk, and both the level assigned to the high and medium priority causes generally match its location on the Ishikawa diagram. This situation indicates that it is clear what actions must be taken to control this equipment's risk, those related to the electrical supply, its use, the battery's charge, and electrodes. In the case of the volumetric ventilator, the highest priority causes are related to the operator's handling of equipment components, particularly the filter and the power cable. When complementing the Ishikawa diagram analysis, a situation similar to the one presented 
in the vital signs monitor can be seen, a training and support material problem is detected.

In general, the found results contrast with those of other studies that used the FMEA method in the ICU, where the failures with the highest priority related to alarms of the ventilation device ${ }^{17}$ or increased rates of internal infection due to the inadequate operation of the medical equipment ${ }^{18}$ are reported. It is important to note that the FMEA method's application in each case depends on the quantity and quality of the information collected and that the value of the results will be maintained in direct relation to its updating.

The limitations of this article are oriented to the data and its scope. Information from general and non-formal sources was used instead of a specific source such as an ICU of a particular hospital or a health system. Consequently, the results show generalized trends in the community regarding the causes of failures. Therefore, the information obtained from the analysis can be a starting point to develop action plans that can be improved by providing feedback with the particular ICU's specific data under consideration.

The FMEA method is a comprehensive tool for developing risk management programs; what is proposed is to use some of its components to build support elements for these programs, focusing on specific aspects, such as the operation of the medical device. In a previous approach, information was considered to assign priorities related to functioning. ${ }^{19}$ Then, using the concepts of severity, occurrence, and detection, an analysis of failures is complemented, attending to the causes and giving them a complete management approach. In this case, we used these concepts as a basis and adapted them by defining them in the context of the information presented in the forums. More advanced work in this field, incorporating information derived from tools such as orders or service logs, is in process.

\section{CONCLUSIONS}

The development of risk management methodologies that aim to monitor and solve potential hazard situations in critical areas is valuable to the health technology management program. The FMEA method showed a solid basis for the risk assessment processes, and its application to the ICU medical technology allowed the creation of the evidence supporting the decision-making process concerning strategic solutions to guarantee patient safety.

\section{CONFLICT OF INTEREST}

The authors declare that they have no conflict of interest.

\section{REFERENCES}

1. Raffin TA. Ethical concerns in managing critically ill patients. In Critical Care Medicine: Principles of Diagnosis and Management in the Adult, $2 \mathrm{dn}$ edition. St. Louis (MO): Mosby; 2002:1447-1464.

2. da Silva G, da Silva R, Ferreira M. Technologies in intensive care: causes of adverse events an implications to nursing. Rev Bras Enferm. 2016;69(5):915-923. http://dx.doi.org/10.1590/0034-7167.2016690505

3. Thomas A, Galvin I. Patient safety incidents associated with equipment in critical care: a review of reports in the UK National Patient Safety Agency. Anaesthesia 2008;63(11):1193-1197. https://doi. org/10.1111/j.1365-2044.2008.05607.x

4. Vincent C, Taylor-Adams S, Stanhope N. Framework for analyzing risk and safety in clinical medicine BMJ 1998;316(7138):1154-1157. https://doi.org/10.1136/ bmj.316.7138.1154

5. Martinez-Licona F, Azpiroz-Leehan J, Cadena-Méndez M, Coronel J, Rechy E. Hospital technological failure classification. In III Cong. Colombiano de Bioing. Ing. Biomédica, Pereira, Colombia, 2008:1-4 (In Spanish).

6. Stamatis DH. Failure Mode and Effect Analysis FMEA from Theory to Execution. Milwaukee (WI): ASQ Quality Press; 2003.

7. Faiella G, Parand A, Franklin BD, Chana P, Cesarelli M, Stanton NA. Expanding healthcare failure mode and effect analysis: A composite proactive risk analysis approach. Rel Eng Syst Saf. 2018;169:117-126. https:// doi.org/10.1016/j.ress.2017.08.003

8. Spath PL. Using failure model and effects analysis to improve patient safety. AORN J 2003;78(1):15-37. https://doi.org/10.1016/S0001-2092(06)61343-4

9. Manrique-Rodríguez S, Sánchez-Galindo A, LópezHerce J, Calleja-Hernández M, Iglesias-Peinado I, 
Carrillo-Álvarez A, Fernández-Llamazares C. Risks in the implementation and use of smart pumps in a pediatric intensive care unit: application of the failure mode and effect analysis. Int J Technol Assess Health Care 2014;30(2):210-217. https://doi.org/10.1017/ S0266462314000051

10.Chadwick L, Fallon E. Evaluation and critique of Healthcare Failure Mode and Effect Analysis applied in a radiotherapy case study. Hum. Factors Ergonomics Manuf. Serv. Ind. 2013;23(2):116-127. https://doi. org/10.1002/hfm.20302

11.Jiang Y, Jiang H, Ding S, Liu Q. Application of failure mode and effects analysis in a clinical chemistry laboratory. Clin Chim Acta 2015;448(25):80-85. https:// doi.org/10.1016/j.cca.2015.06.016

12. Mikulak RJ, Mc Dermott R, Beauregard M. The basics of FMEA, 2nd edition. New York (NY): CRC Press; 2009.

13. Luca L. A new model of Ishikawa diagram for quality assessment. In IOP Conf. Ser. :Mater. Sci., Kozani, Grecia; 2016:1-6. https://doi.org/10.1088/1757-899X/161/1/012099

14.Norma Oficial Mexicana NOM-025-SSA3-2013, para la organización y funcionamiento de las unidades de cuidados intensivos On Diario Oficial de la Federación; 2013. Available at: https://www.gob.mx/cms/uploads/ attachment/file/35891/NOM-025-SSA3- 2013.pdf
15. Core medical equipment. On World Health Organization; 2011. Available at: https://apps.who.int/iris/ handle/10665/95788

16.Wåhlin I, Ek AC, Lindgren M, Geijer S, Årestedt K. Development and validation of an ICU-specific pressure injury risk assessment scale. Scandinavian Journal of Caring Sciences 2020. https://doi.org/10.1111/ scs.12891

17.Asefzadeh S, Yarmohammadian M, Atighechian G. Clinical risk assessment in intensive care unit. International Journal of Preventive Medicine 2013;4(5): 592-598. https://www.ncbi.nlm.nih.gov/pmc/articles/ PMC3733191/

18. Homauni A, Zargar S, Hazrati E, Markazi N. Intensive care unit risk assessment: a systematic review. Iranian Journal of Public Health 2020;49(8):1422-1431. https://doi.org/10.18502/ijph.v49i8.3865

19.Perez-Ramos S, Martinez-Licona F. Failure Analysis to Evaluate the Functioning Quality of Medical Devices: Case of the Volumetric Ventilator in the Adult Intensive Care Area. In Proceedings of the Nacional Congress of Biomedical Engineering, Mexico 2020;7(1):351-358, 2020 (In Spanish). http://memorias.somib.org.mx/ index.php/memorias/article/view/783 